\title{
Nonpsychotropic plant cannabinoids, cannabidivarin (CBDV) and cannabidiol $(C B D)$, activate and desensitize transient receptor potential vanilloid 1 (TRPV1) channels in vitro: potential for the treatment of neuronal hyperexcitability
}

Article

Accepted Version

Iannotti, F. A., Hill, C. L., Leo, A., Alhusaini, A., Soubrane, C., Mazzarella, E., Russo, E., Whalley, B. J., Di Marzo, V. and Stephens, G. J. (2014) Nonpsychotropic plant cannabinoids, cannabidivarin (CBDV) and cannabidiol (CBD), activate and desensitize transient receptor potential vanilloid 1 (TRPV1) channels in vitro: potential for the treatment of neuronal hyperexcitability. ACS Chemical Neuroscience, 5 (11). pp. 1131-1141. ISSN 1948-7193 doi: https://doi.org/10.1021/cn5000524 Available at https://centaur.reading.ac.uk/37398/

It is advisable to refer to the publisher's version if you intend to cite from the work. See Guidance on citing. 
All outputs in CentAUR are protected by Intellectual Property Rights law, including copyright law. Copyright and IPR is retained by the creators or other copyright holders. Terms and conditions for use of this material are defined in the End User Agreement.

\section{$\underline{\text { www.reading.ac.uk/centaur }}$}

\section{CentAUR}

Central Archive at the University of Reading

Reading's research outputs online 


\section{Nonpsychotropic plant cannabinoids, cannabidivarin (CBDV) and cannabidiol $(C B D)$, activate and desensitize transient receptor potential vanilloid 1 (TRPV1) channels in vitro: potential for the treatment of neuronal hyperexcitability}

Article

Accepted Version

paper

lannotti, F. A., Hill, C. L., Leo, A., Alhusaini, A., Soubrane, C. , Mazzarella, E. , Russo, E. , Whalley, B. J., Di Marzo, V. and Stephens, G. J. (2014) Nonpsychotropic plant cannabinoids, cannabidivarin (CBDV) and cannabidiol (CBD), activate and desensitize transient receptor potential vanilloid 1 (TRPV1) channels in vitro: potential for the treatment of neuronal hyperexcitability. ACS Chemical Neuroscience, 5 (11). pp. 1131-1141. ISSN 1948-7193 doi: 10.1021/cn5000524 Available at http://centaur.reading.ac.uk/37398/

It is advisable to refer to the publisher's version if you intend to cite from the work.

To link to this article DOI: http://dx.doi.org/10.1021/cn5000524

Publisher: ACS Publications 
All outputs in CentAUR are protected by Intellectual Property Rights law, including copyright law. Copyright and IPR is retained by the creators or other copyright holders. Terms and conditions for use of this material are defined in the End User Agreement.

\section{www.reading.ac.uk/centaur}

\section{CentAUR}

Central Archive at the University of Reading

Reading's research outputs online 


\section{Nonpsychotropic Plant Cannabinoids, Cannabidivarin (CBDV) and Cannabidiol (CBD), Activate and Desensitize Transient Receptor Potential Vanilloid 1 (TRPV1) Channels in Vitro: Potential for the Treatment of Neuronal Hyperexcitability}

Fabio Arturo Iannotti, ${ }^{\dagger}$ Charlotte L. Hill, ${ }^{\ddagger}$ Antonio Leo, ${ }^{\ddagger}$, Ahlam Alhusaini, ${ }^{\ddagger}$ Camille Soubrane, ${ }^{\ddagger}$ Enrico Mazzarella, ${ }^{\dagger}$ Emilio Russo, ${ }^{\|}$Benjamin J. Whalley, ${ }^{\ddagger}$ Vincenzo Di Marzo, ${ }^{*}{ }^{\dagger}$ and Gary J. Stephens ${ }^{*}, \ddagger$

${ }^{\dagger}$ Endocannabinoid Research Group, Institute of Biomolecular Chemistry (ICB), National Council of Research (CNR), 80078

Pozzuoli (NA) Italy

"School of Pharmacy, University of Reading, Whiteknights, Reading, RG6 6AJ, United Kingdom

Science of Health Department, ${ }^{\S}$ School of Pharmacy and "School of Medicine, University of Catanzaro, 88100 Catanzaro, Italy

Supporting Information

ABSTRACT: Epilepsy is the most common neurological disorder, with over 50 million people worldwide affected. Recent evidence suggests that the transient receptor potential cation channel subfamily V member 1 (TRPV1) may contribute to the onset and progression of some forms of epilepsy. Since the two nonpsychotropic cannabinoids cannabidivarin (CBDV) and cannabidiol (CBD) exert anticonvulsant activity in vivo and produce TRPV1-mediated intracellular calcium elevation in vitro, we evaluated the effects of these two compounds on TRPV1 channel activation and desensitization and in an in vitro model of epileptiform activity. Patch clamp analysis in transfected HEK293 cells demonstrated that CBD and CBDV dose-dependently activate and rapidly desensitize TRPV1, as well as TRP channels of subfamily $\mathrm{V}$ type 2 (TRPV2) and subfamily A type 1 (TRPA1). TRPV1 and TRPV2 transcripts were shown to be expressed in rat hippocampal tissue. When tested on epileptiform neuronal spike activity in hippocampal brain slices exposed to a $\mathrm{Mg}^{2+}$-free solution using multielectrode arrays (MEAs), CBDV reduced both epileptiform burst amplitude and duration. The prototypical TRPV1 agonist, capsaicin, produced similar, although not identical effects. Capsaicin, but not CBDV, effects on burst amplitude were reversed by IRTX, a selective TRPV1 antagonist. These data suggest that CBDV antiepileptiform effects in the $\mathrm{Mg}^{2+}$-free model are not uniquely mediated via activation of TRPV1. However, TRPV1 was strongly phosphorylated (and hence likely sensitized) in $\mathrm{Mg}^{2+}$-free solution-treated hippocampal tissue, and both capsaicin and CBDV caused TRPV1 dephosphorylation, consistent with TRPV1 desensitization. We propose that CBDV effects on TRP channels should be studied further in different in vitro and in vivo models of epilepsy.

KEYWORDS: Cannabinoid, TRP channels, epilepsy, patch clamp, multi electrode arrays (MEAs)
$\mathrm{E}$ pilepsies represent a subset of neuronal dysrhythmias caused by, among others, defects in membrane excitability, which leads to the aberrant synchronization of neural networks. This disturbance in the electrical activity is characterized by a long-term risk of recurrent seizures. ${ }^{1}$ The incidence of epilepsy in the developed world is remarkably high. The number of people affected worldwide is more than 50 million $(>1.0 \%)$, with an incidence of 20-70 new cases/10 000 individuals. The majority of new cases occur most frequently in infants and the elderly. ${ }^{2,3}$ To date, epilepsy cannot be cured; except for a small minority of cases that can be approached surgically, the treatment of epilepsy is largely based on the use of antiepileptic drugs (AEDs) to achieve symptomatic seizure control. Currently, about $70 \%$ of people with epilepsy can be successfully treated by the large spectrum of AEDs available, each with discrete pharmacokinetics, spectrum of antiepileptic activity, and toxicity. The molecular targets of most of these AEDs are restricted to voltage-gated $\mathrm{Na}^{+}, \mathrm{K}^{+}$, and $\mathrm{Ca}^{2+}$ channels, $\mathrm{GABA}_{\mathrm{A}}$ receptors, the GAT-1 GABA transporter, glutamate, GABA transaminase, and synaptic proteins involved in neurotransmitter release, such as SV2A. ${ }^{2}$ In general, current AEDs work by changing the levels of neurotransmitters in the brain that regulate electrical impulses. However, in spite of such a wide therapeutic armamentarium, 30\% of epileptic patients

Special Issue: TRPs as Probes and Medications for CNS Disorders

Received: March 9, 2014

Revised: June 17, 2014 
still do not receive satisfactory seizure control due to their unresponsiveness to AEDs. ${ }^{2}$ Recently, we have shown that two of the nonpsychoactive constituents of Cannabis sativa, cannabidiol (CBD) and cannabidivarin (CBDV), which are unrelated to current AEDs, are emerging candidates with the potential to reduce seizures. ${ }^{4-8}$ However, the exact molecular mechanism of the anticonvulsant action of these two phytocannabinoids, which are currently undergoing clinical investigation, remains to be elucidated.

Transient receptor potential (TRP) channels are a large subfamily of ion channels characterized by weak voltage sensitivity and a nonselective permeability to monovalent and divalent cations including $\mathrm{Mg}^{2+}, \mathrm{Ca}^{2+}$, and $\mathrm{Na}^{+}$. TRP channels are expressed throughout the body and their activation in neurons serves to transduce chemical or physical stimuli into nerve impulses to transmit to the brain. TRPV1, also known as the capsaicin receptor or vanilloid receptor 1, was the first member of the TRPV subfamily to be discovered and cloned. ${ }^{9}$ We report, through the use of patch clamp analysis performed in transfected HEK293 cells, that both CBD and CBDV dosedependently activate and rapidly desensitize TRPV1, as well as TRPV2 and TRPA1, channels. Measurement of hippocampal slice neuronal activity exposed to $\mathrm{Mg}^{2+}$-free solution using multielectrode arrays (MEAs) revealed that $\mathrm{CBDV}$, similar to capsaicin, significantly reduces amplitude of epileptiform bursts. Further experiments indicated that CBDV effects in this in vitro model of epileptogenic activity may not be uniquely mediated by TRPV1 channels. However, capsaicin and CBDV both caused TRPV1 dephosphorylation and, consequentially, may cause channel desensitization, suggesting that CBDV effects on TRP channels should be studied further in different models of epilepsy.

\section{RESULTS AND DISCUSSION}

TRPV1, TRPV2, and TRPA1 Channels Are Activated and Rapidly Desensitized by CBDV and CBD: Whole-Cell Patch Clamp Analysis. Some phytocannabinoids have the potential to address the unmet clinical need associated with a range of treatment-resistant epilepsies; ${ }^{6}$ we have shown that both CBD and CBDV are anticonvulsant in a range of in vitro and in vivo models ${ }^{4-8}$ and that $\mathrm{CBDV}$ can suppress pentylenetetrazole (PTZ)-induced increases in epilepsy-related gene expression. ${ }^{10}$ However, despite this preclinical and clinical promise, the mechanisms whereby CBD or CBDV exert their anticonvulsant effects have not been elucidated. CBD and CBDV have extremely low, physiologically irrelevant, affinity for cannabinoid $\mathrm{CB}_{1}$ receptors, and both pure $\mathrm{CBDV}$ and an anticonvulsant $\mathrm{CBDV}$-enriched extract have been shown to act in a $\mathrm{CB}_{1}$-independent manner. ${ }^{8} \mathrm{CBD}$ has a number of different potential anticonvulsant molecular mechanisms. ${ }^{5,11}$ Moreover, $\mathrm{CBD}$, and the related phytocannabinoid cannibigerol (CBG) can block voltage-gated $\mathrm{Na}^{+}$channels in vitro but this action does not contribute to their anticonvulsant activity, as CBG had no effect in the PTZ model of seizure. ${ }^{12}$ Previous studies have suggested that TRP channels are molecular targets for phytocannabinoids. We and others have shown that $\mathrm{CBD}$ activates TRPV1, TRPV2, and TRPA1, ${ }^{13-16}$ while CBDV also acts at these TRP isoforms, in some cases with slightly lower potency. ${ }^{15}$ Here, we performed patch clamp studies with CBD and CBDV in HEK293 cells transiently overexpressing rat recombinant TRPV1 channels. A relatively selective agonist of TRPV1 channels, capsaicin, at the concentrations of 1 and 10 $\mu \mathrm{M}$, evoked a bidirectional current across the voltage range
$(-80$ to $+80 \mathrm{mV})$, with a reversal potential near $0 \mathrm{mV}$ (Figure 1a). Such data is consistent with the widely reported

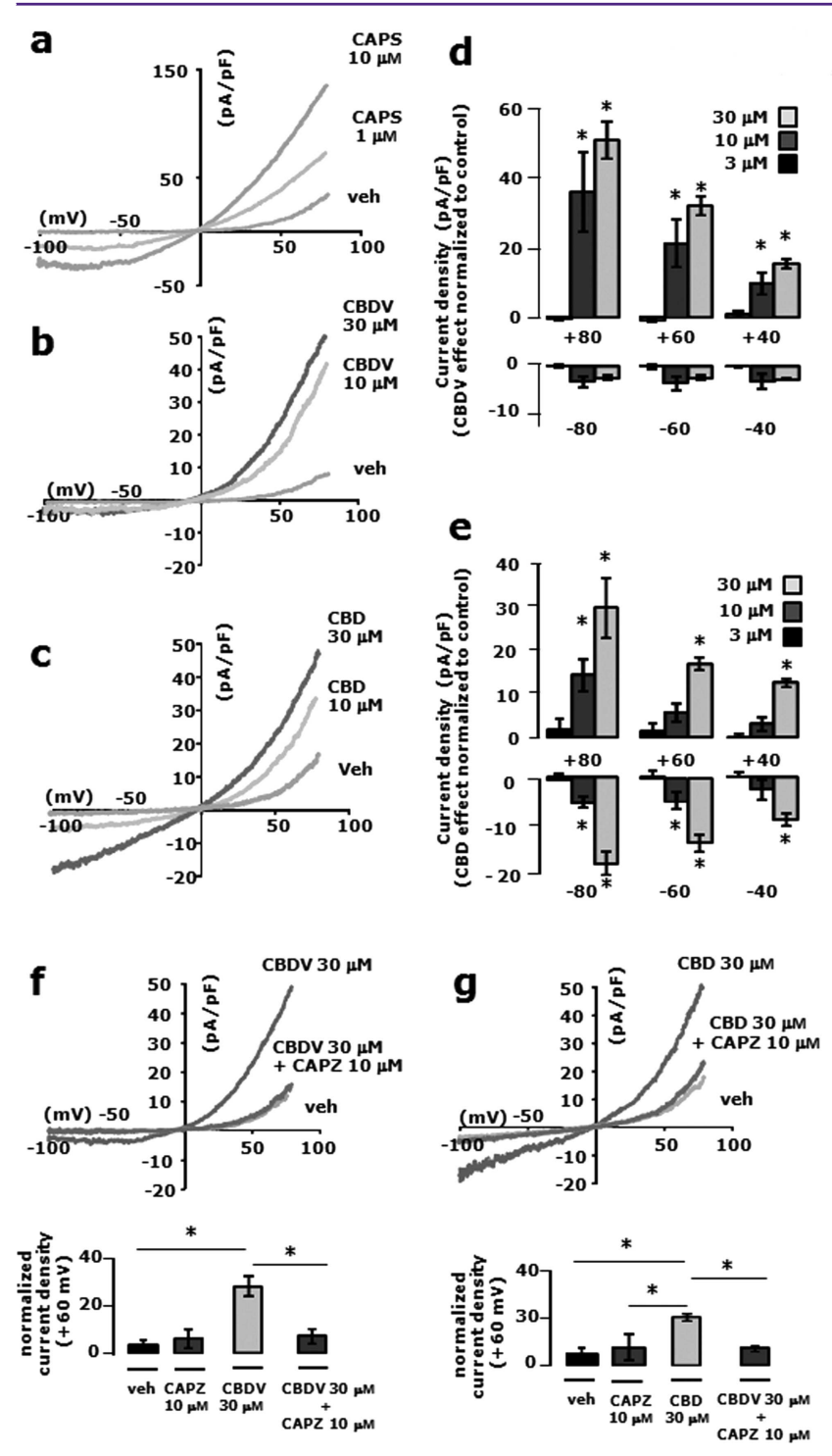

Figure 1. Effects of CBDV and CBD in HEK293 transiently expressing rat TRPV1 channels: (a) Representative traces of capsaicin (CAPS; 1$10 \mu \mathrm{M}$ ) effects in HEK293 expressing rat TRPV1 channels. (b) CBDV $(10-30 \mu \mathrm{M})$ and (c) CBD $(10-30 \mu \mathrm{M})$ effects on TRPV1 channel current density compared to control (vehicle, DMSO). (d) Effects of CBDV $(3-30 \mu \mathrm{M})$ and (e) CBD $(3-30 \mu \mathrm{M})$ on TRPV1 current expressed as current density relative to control at a distinct range of membrane potentials. (f) Representative traces (upper panel) showing capsazepine (CAPZ; $10 \mu \mathrm{M})$ block of CBDV $(30 \mu \mathrm{M})$ and $(\mathrm{g}) \mathrm{CBD}$ $(30 \mu \mathrm{M})$ effect on TRPV1 currents. The bottom panels in $(\mathrm{f})$ and $(\mathrm{g})$ show quantified data. Each bar is the mean \pm SEM of at least four separate determinations. ${ }^{*} p<0.05$ vs vehicle (Student's $t$ test).

nonselective cationic current activated by TRP isoforms. Compared to vehicle (DMSO), CBDV or CBD (3, 10, and $30 \mu \mathrm{M})$ dose-dependently evoked a TRPV1 channel-mediated current characterized by a bidirectional current similar to capsaicin (Figure $1 \mathrm{~b}$ and $\mathrm{c}$ ). For $\mathrm{CBDV}$ and $\mathrm{CBD}$, the current reversal potential point was close to $0 \mathrm{mV}$ (Figure $1 \mathrm{~b}$ and $\mathrm{c}$ ). The normalized current density values for CBDV and CBD were estimated by extrapolating membrane potential values at $\pm 80, \pm 60$, and $\pm 40 \mathrm{mV}$ (Figure $1 \mathrm{~d}$ and e). The TRPV1 

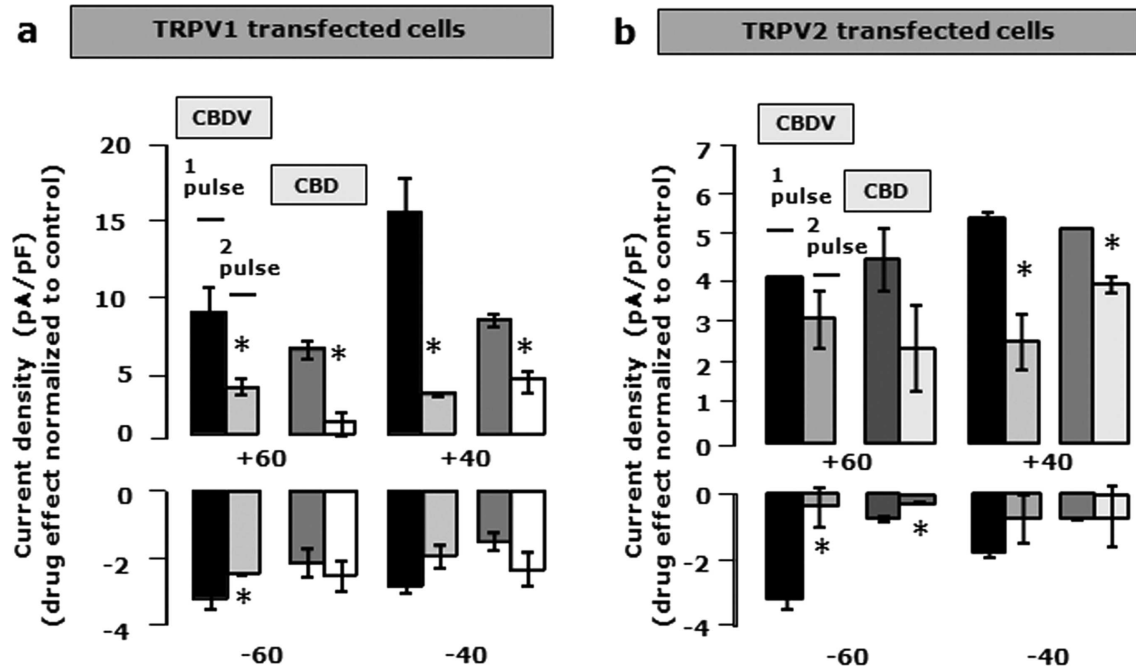

Figure 2. Desensitization of rat TRPV1 and TRPV2 channels by CBDV and CBD: Bar graphs showing current density values upon the repeated exposure to CBDV $(10 \mu \mathrm{M})$ and CBD $(10 \mu \mathrm{M})$ in HEK293 cells transiently expressing TRPV1 (a) and TRPV2 (b) channels. The second pulse was given 5-10 min after the first one. Each bar is the mean \pm SEM of at least four separate determinations. ${ }^{*} p<0.05$ vs the first pulse.

antagonist capsazepine (CAPZ; $10 \mu \mathrm{M})$ abolished the effects of CBDV and of CBD, confirming that these currents were mediated by TRPV1 (Figure If and g). Capszepine effect was also quantified by normalizing current density values relative to control (Figure 1f and g; bottom panel). In this system, recombinant TRPV1 channels are rapidly desensitized upon the second exposure to $1 \mu \mathrm{M}$ capsaicin or $10 \mu \mathrm{M}$ CBDV and CBD (Figure 2a).

We next tested the effects of CBDV and $\mathrm{CBD}$ on rat recombinant TRPV2 channels. Similar to the effects observed for TRPV1 channels and when compared to vehicle, CBDV and $\mathrm{CBD}(3,10$, and $30 \mu \mathrm{M})$ dose-dependently evoked a bidirectional current across the voltage range $(-80$ to $+80 \mathrm{mV})$ with a reversal potential near to $0 \mathrm{mV}$ (Figure $3 \mathrm{a}-\mathrm{d}$ ). CBDVand $\mathrm{CBD}$-evoked currents were completely blocked by ruthenium red ( $R R ; 10 \mu \mathrm{M})$, a widely used TRP channel blocker, (Figure $3 e$ and f). TRPV2 channels were rapidly desensitized upon second exposure to $10 \mu \mathrm{M} \mathrm{CBD}$ and CBDV (Figure 2b).

Given that CBD and CBDV have previously been proposed to activate TRPAl channels, ${ }^{14}$ we also tested their effects on rat TRPA1, using mustard oil (MO), a selective agonist of TRPA1 channels as a positive control. As shown in Figure 4a, MO (100 $\mu \mathrm{M})$ evoked a large TRPA1-mediated bidirectional current which reversed direction around $0 \mathrm{mV}$. CBDV and CBD $(3,10$, and $30 \mu \mathrm{M})$ dose-dependently evoked a TRPAl current, as reflected by the bidirectional increased current density (Figure $4 \mathrm{~b}-\mathrm{e})$. RR $(10 \mu \mathrm{M})$ abolished the effects of both CBDV and CBD (Figure $4 \mathrm{f}$ and $\mathrm{g}$ ), consistent with effects on a TRPmediated conductance. As a negative control for this set of experiments, as well as of those described above, CBDV and CBD $(10 \mu \mathrm{M})$ were both applied to untransfected HEK293 cells and shown not to evoke any detectable current above vehicle effects (Supporting Information Figure 1).

Overall, we have extended our previous intracellular $\mathrm{Ca}^{2+}$ studies ${ }^{14,15}$ by using the patch clamp technique to provide evidence for direct interaction between $\mathrm{CBD}$ and CBDV with rat TRPV1, TRPV2 and TRPA1 channels. As with intracellular $\mathrm{Ca}^{2+}$ experiments, ${ }^{14,15} \mathrm{CBD}$ and $\mathrm{CBDV}$ can act at low micromolar concentrations to activate TRPV1, TRPV2 and TRPA1 cation channels. Most interestingly, CBD and CBDV activation was associated with channel desensitization on subsequent application. TRPV1, as with other TRP channels, is widely reported to be activated and rapidly desensitized following prolonged exposure to canonical agonists such as capsaicin, ${ }^{17-19}$ rendering them refractory to further stimulation. This desensitization is thought to underlie the paradoxically analgesic effect of TRPV1 agonists ${ }^{17}$ and may explain how TRP activation could reduce neuronal activity in diseases of neuronal hyperexcitability, such as epilepsy.

TRPV1 and TRPV2 Expression in Rat Hippocampal Tissue. We next investigated the expression of transcripts encoding for TRPV1, TRPV2 and TRPA1 channels in the rat hippocampus by means of qPCR experiments, performed with gene-selective primers (Supporting Information Table 1). These data revealed that the genes encoding both TRPV1 and TRPV2 are expressed in both sides of the adult rat hippocampus (Figure 5a). By contrast, and in agreement with the prevailing literature, ${ }^{20}$ TRPA1 mRNA expression was barely detectable. In comparison to TRPV1 mRNA expression, TRPV2 was expressed at significantly higher levels, while TRPA1 was expressed at significantly lower levels. In keeping with the observed TRPV1 and TRPV2 mRNA expression profile, Western blot analysis revealed that TRPV2 is more strongly expressed than TRPV1 in rat hippocampal tissue (Figure 5b).

TRPV1 channels are predominantly expressed in nociceptive neurons of the peripheral nervous system, where their activation leads to the transmission and modulation of painrelated conditions, such as inflammatory thermal hyperalgesia and neuropathic pain. ${ }^{21}$ TRPV1 expression in the CNS is more controversial, although several studies have reported TRPV1 expression in mammalian brain regions including the hypothalamus, cerebellum, cerebral cortex, striatum, midbrain, hippocampus, and substantia nigra. ${ }^{22-27}$ To gain further insight into the localization of TRPV1 channels in hippocampal neurons, immunofluorescence experiments on rat brain cryosections revealed that, in the CA1 region, TRPV1 immunofluorescence was partly associated with vGLUT (vesicular glutamate transporter 1), a widely used marker of glutamatergic nerve terminals ${ }^{28}$ (Figure $6 a-d$ ). By contrast, TRPV1 immunoreactivity appeared to mostly not associate 


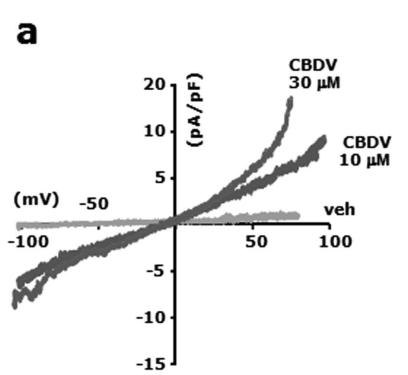

b
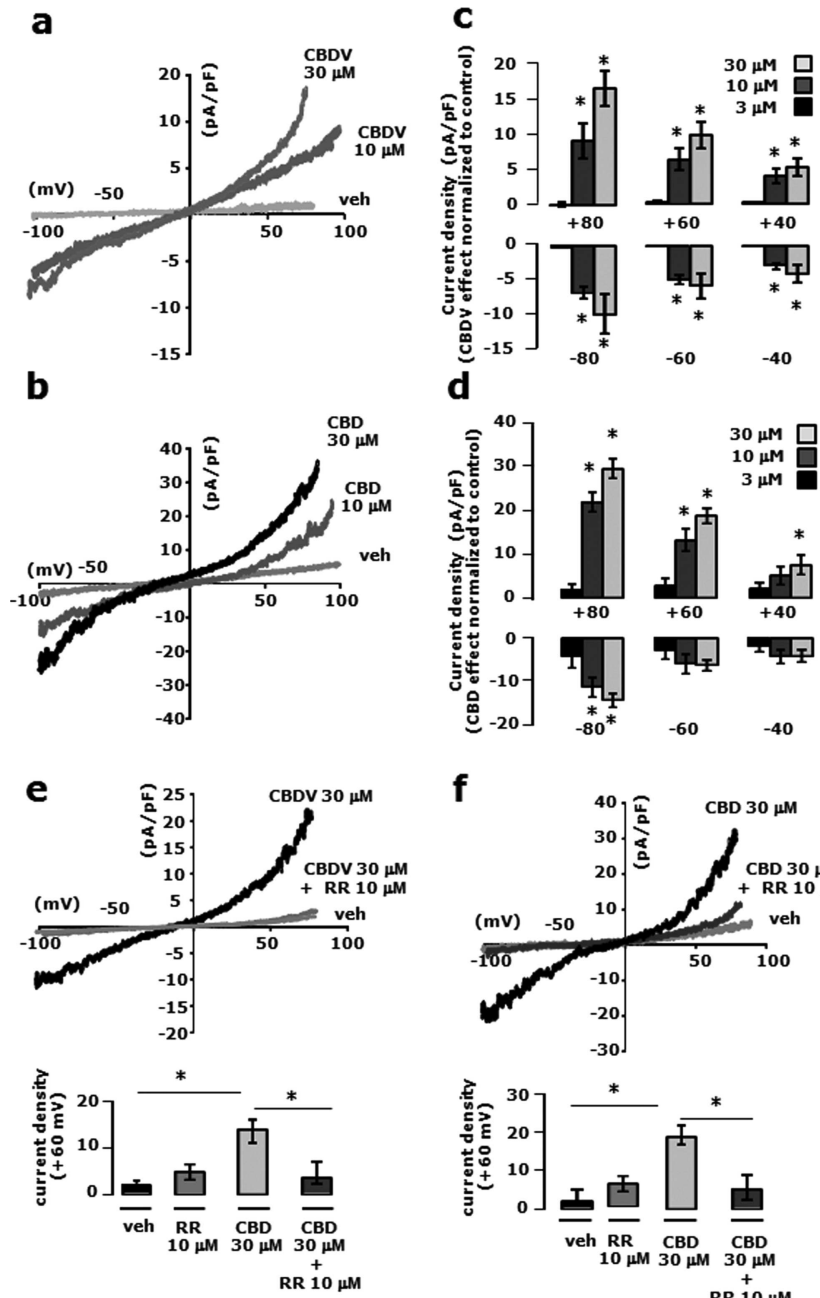

d

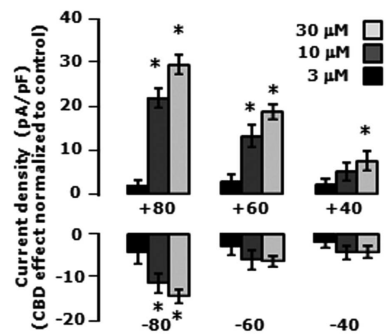

f
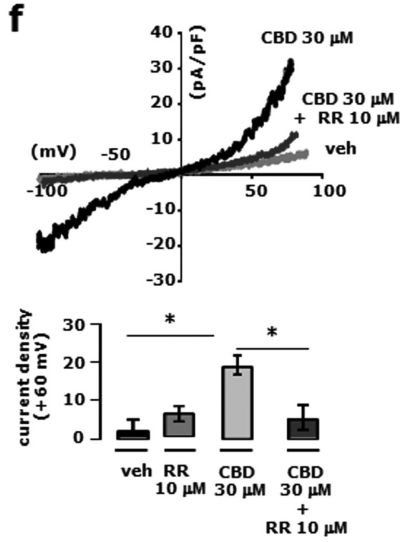

Figure 3. Effects of CBDV and CBD in HEK293 transiently expressing rat TRPV2 channels: Representative traces showing (a) CBDV (10$30 \mu \mathrm{M})$ and (b) CBD $(10-30 \mu \mathrm{M})$ effects on rat TRPV2 current density compared to control (vehicle). Effects of (c) CBDV (3-30 $\mu \mathrm{M})$ and (d) CBD $(3-30 \mu \mathrm{M})$ on TRPV2 current expressed as current density at a distinct range of membrane potentials. Representative traces (upper panel) showing ruthenium red (RR; 10 $\mu \mathrm{M})$ block of (e) CBDV $(30 \mu \mathrm{M})$ and (f) CBDV $(30 \mu \mathrm{M})$ effects on TRPV2 currents. The bottom panels in (f) and ( $g$ ) show the quantified data. Each bar is the mean \pm SEM of at least four separate determinations. $* p<0.05$ vs vehicle.

with vGAT (antivesicular GABA transporter), a common marker of GABAergic neurons ${ }^{29}$ (Figure $6 \mathrm{e}-\mathrm{h}$ ). These experiments suggest that TRPV1 is ideally located in the hippocampal CA1 region to modulate also excitatory glutamate release and signaling.

TRPV2 expression has been reported in the brain, ${ }^{30}$ but the exact localization and function of this subclass of TRPV channels have not been elucidated. Our results reveal that the TRPV2 signal does not appear to be associated with either presynaptic vGLUT or vGAT (Figure $6 \mathrm{i}-\mathrm{o}$ ), indicating that TRPV2 channels are most likely expressed postsynaptically. Interestingly, we also found that TRPV2 is robustly expressed in what appears to be, morphologically, non-neuronal cells (Figure 6o, white arrows). It remains to be confirmed if these cells are astrocytes, which have been recently reported to express TRPV2. ${ }^{31}$ a

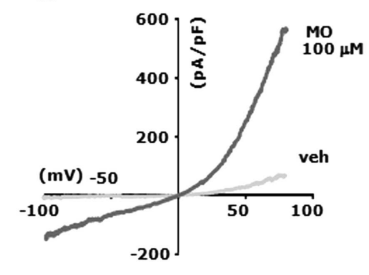

b
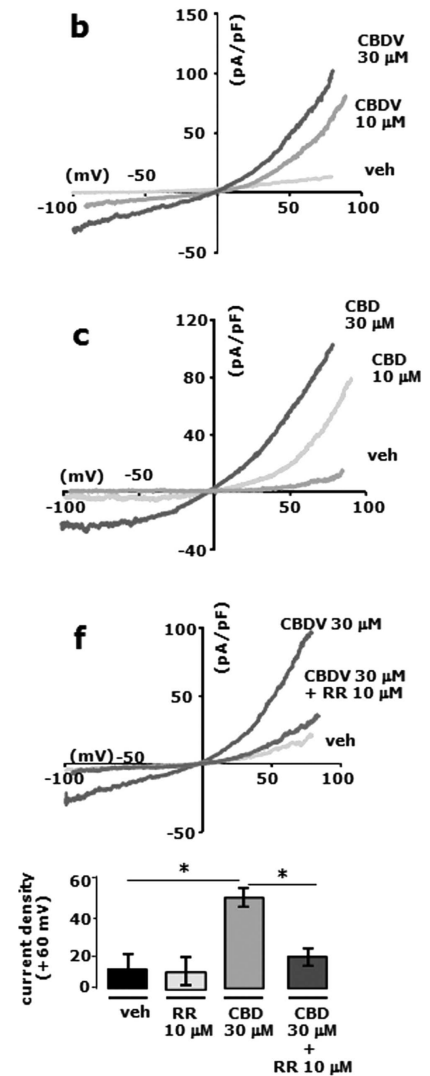
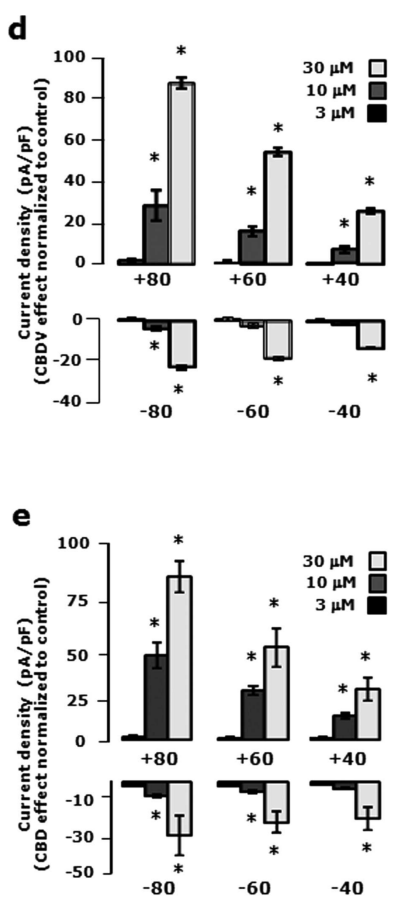

g
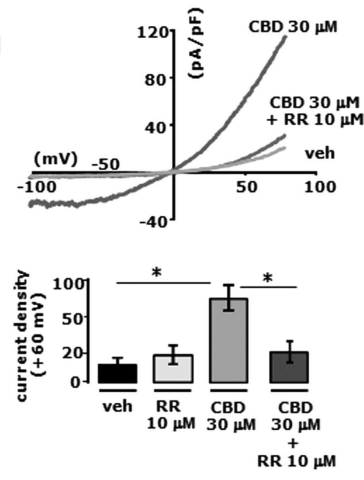

Figure 4. Effects of CBDV and CBD in HEK293 transiently expressing rat TRPA1 channels: (a) Representative traces showing mustard oil $(\mathrm{MO} ; 100 \mu \mathrm{M})$ effects in rat HEK293 TRPA1 current density compared to control (vehicle). (b) CBDV (10-30 $\mu \mathrm{M}$ ) and (c) CBD $(10-30 \mu \mathrm{M})$ effects on TRPA1 current density compared to control (vehicle). Effects of (d) CBDV and (e) CBD on TRPA1 current expressed as current density at a distinct range of membrane potentials. Representative traces (upper panel) showing ruthenium red (RR; $10 \mu \mathrm{M})$ block of (f) CBDV $(30 \mu \mathrm{M})$ and (g) CBD $(30 \mu \mathrm{M})$ effects on TRPV1 currents. The bottom panels in (f) and (g) show the quantified data. Each bar is the mean \pm SEM of at least four separate determinations. $* p<0.05$ vs vehicle.

We next focused on TRPV1 channels and their presynaptic role in determining neuronal excitability. Importantly, there is the possibility that low levels of TRPV1 reported in the aforementioned studies, are substantially upregulated in conditions of hyperexcitability, such as epilepsy. Thus, recent studies have shown that changes in TRPV1 expression and activity facilitate epileptogenesis in patients affected by mesial temporal lobe epilepsy ${ }^{32}$ and that TRPV1 activation by anandamide increases excitatory circuit activity in the synaptically reorganized dentate gyrus. ${ }^{33}$ A more recent study demonstrated that TRPV1 channels may be involved in the development of electrical and PTZ-induced kindling. ${ }^{34}$ On the other hand, PTZ-induced clonic seizures were reported to be reduced in TRPV1 knockout mice. ${ }^{35}$ By contrast, to the best of 


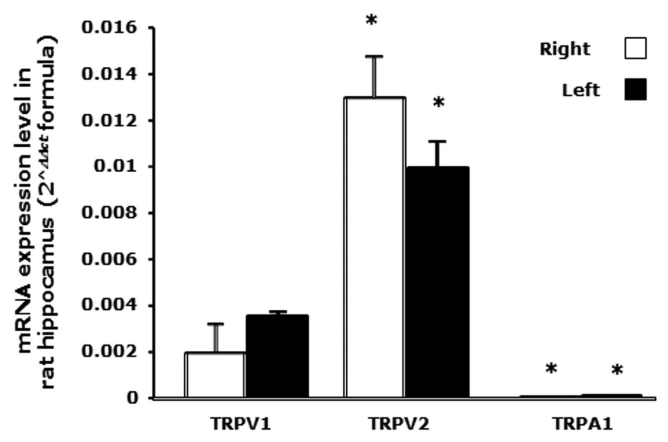

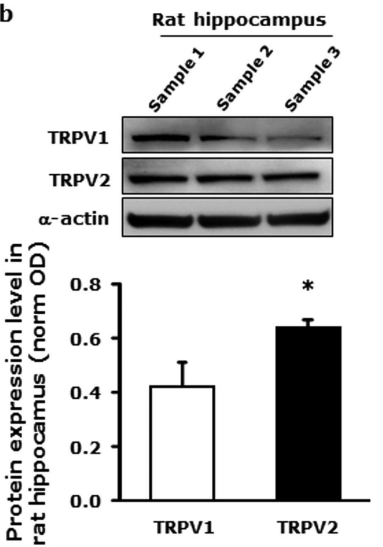

Figure 5. Expression of TRPV1, TRPV2, and TRPA1 transcripts in rat hippocampal tissue: (a) Transcripts levels of TRPV1, TRPV2, and TRPA1 genes were evaluated in ipsalateral and contralateral sides of the rat hippocampus by qPCR analysis. The data are expressed by calculating the $2^{\Delta c t}$ formula (see Methods). Each bar is the mean \pm SEM of at least four separate determinations. ${ }^{*} p<0.05$ vs TRPV1 mRNA expression level. (b) Western blot analysis of TRPV1 and TRPV2 protein levels in hippocampal tissue. Representative blots showing the approximate molecular mass of each TRPV protein (expressed in $\mathrm{kDa}$ ) (upper) and quantification of the averaged OD values for TRPV1 and TRPV2 normalized to those of $\alpha$ tubulin (lower) are shown. $* P \leq 0.05$ vs TRPV1 protein expression level. Each data point is from at least four independent determinations.
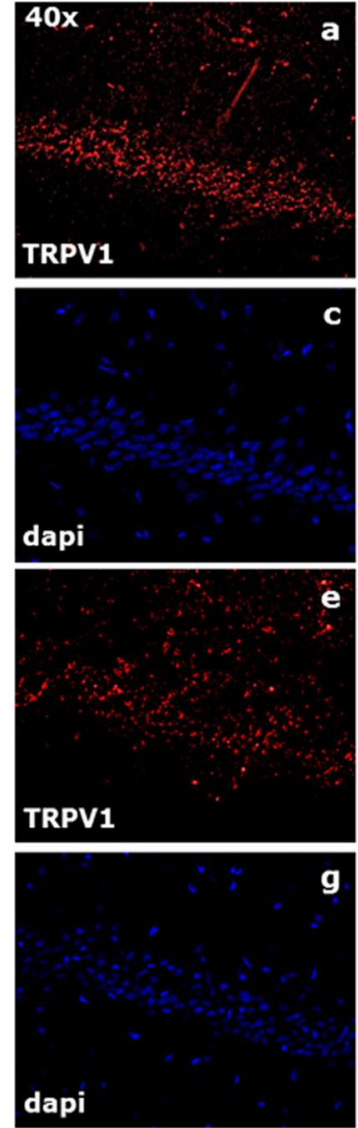
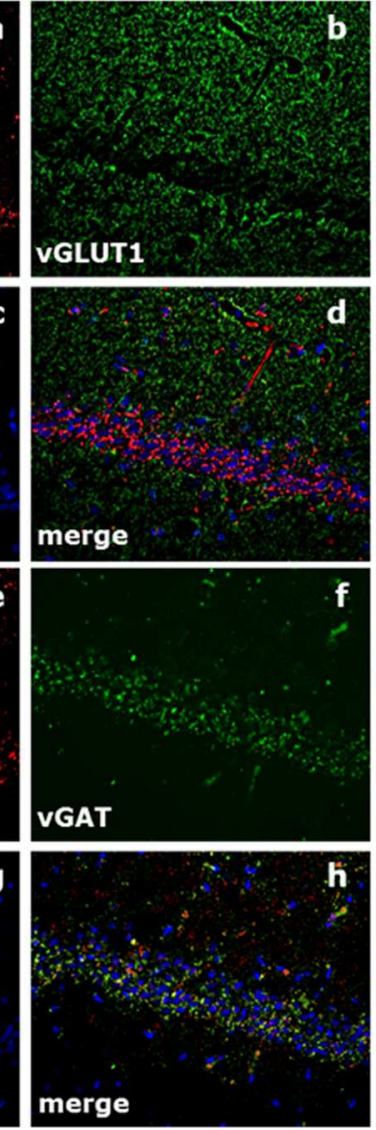
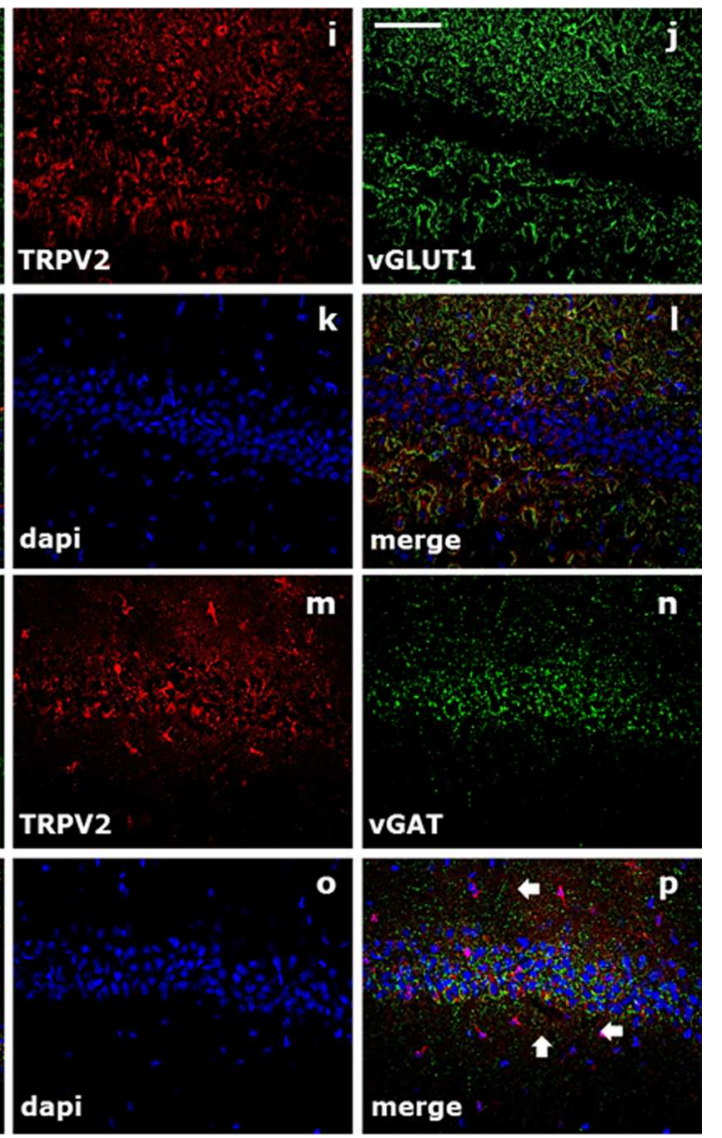

Figure 6. Expression of TRPV1 and TRPV2 in adult rat hippocampal slices. Immunofluorescence images, captured with a 40× objective, of rat hippocampus sagittal cryosections. Red, TRPV1 or TRPV2 immunoreactivity; green, immunoreactivity of (a) vGLUT1 or (b) vGAT; blue, nuclei identified with DAPI. Scale bar, $10 \mu \mathrm{m}$.

our knowledge, there has been no previous report of TRPV2 or TRPA1 being directly associated with models of epileptiform activity and acute seizure. These previous studies provide a putative link between TRPV1 and some forms of seizure, and thus prompted us to investigate here whether phytocannabinoid activation/desensitization of TRPV1 might result in the modulation of neuronal hyperexcitability in vitro.
Capsaicin and CBDV Induce Changes in Hippocampal Neuronal Activity: MEA Analysis. We next extended our studies to electrophysiological field potential recordings in acute hippocampal brain slices using an in vitro model of epileptiform activity. On the basis of our previous work which showed that CBDV can suppress epileptiform activity in hippocampal slices, ${ }^{7}$ and our present findings that (1) CBDV 
exhibits high potency at TRPV1 in patch clamp measurements and (2) there is significant TRPV1 transcript expression in rat hippocampal slices, we compared the effects of CBDV with those of the prototypical TRPV1 agonist, capsaicin, upon epileptiform activity. Specifically, we measured the effect of CBDV and capsaicin on epileptiform activity induced by exposure of acute, hippocampal brain slices to $\mathrm{Mg}^{2+}$-free solution measured using multielectrode array (MEA) electrophysiological field potential recordings. To determine whether acute $\mathrm{CBDV}$ has any effect in this system and to assess the potential contribution of activation/desensitization of TRPV1 channels both to the effects of CBDV and to the epileptiform activity observed, we studied the effects of (1) DMSO (vehicle), (2) $10 \mu \mathrm{M}$ capsaicin, (3) the selective TRPV1 antagonist iodoresiniferatoxin (IRTX; $1 \mu \mathrm{M}$ ), ${ }^{36}$ (4) CBDV (10 $\mu \mathrm{M})$, and (5) CBDV or capsaicin $(10 \mu \mathrm{M})$ in the presence of IRTX $(1 \mu \mathrm{M})$, upon epileptiform activity. Since these experiments were performed in native tissue, as opposed to recombinant HEK293 cells overexpressing only a single TRP channel isoform, IRTX was used since it provides a more selective block of TRPV1 than capsazepine.

Overall, there was a statistically significant effect of drug treatment $(\mathrm{H}(4)=55.62, p \leq 0.0001$; Figure 7a) on epileptiform burst amplitude, which was significantly decreased by (1) capsaicin (71\% of control; $p \leq 0.001 ; n=25$ electrodes from 4 slices), in a manner antagonized by IRTX, (2) CBDV (74\% of control; $p \leq 0.001 ; n=33$ electrodes from 5 slices), and (3) CBDV plus IRTX (69\% of control; $p \leq 0.001 ; n=34$ electrodes from 5 slices). IRTX alone had no significant effect on burst amplitude ( $84 \%$ of control; $n=25$ electrodes from 4 slices), but differed significantly when compared to capsaicin ( $p$ $\leq 0.01)$. There was also a statistically significant difference between the effects of drug treatments on burst duration $(\mathrm{H}(4)$ $=19.79, p=0.0005$; Figure $7 \mathrm{~b}$ ), where burst duration was significantly decreased by CBDV (to $87 \%$ of control; $p \leq 0.001$; $n=33$ electrodes from 5 slices). No other drug treatment produced a significant effect on burst duration. When applied together, IRTX counteracted the effect of CBDV only on duration and not amplitude of neuronal bursts (Figure $7 \mathrm{a}$ and b). Finally, there was no statistically significant difference between effects of drug treatments on burst frequency $(\mathrm{H}(4)=$ 7.69. $p=0.1036$; Figure 7c), although this is an intrinsically variable measure. ${ }^{37}$ Representative traces are shown in Supporting Information Figure 2. These data indicate that while capsaicin and CBDV exert similar effects on epileptiform burst amplitude, unlike capsaicin, CBDV effects on burst amplitude were not sensitive to IRTX. Interestingly, while IRTX did attenuate the CBDV-induced decrease in epileptiform burst duration, capsaicin alone had no effect upon burst duration. Together, these data suggest that CBDV effects in the $\mathrm{Mg}^{2+}$-free model of epileptiform activity are not uniquely mediated via activation of TRPV1.

It has already been established that the phytocannabinoid CBDV exerts antiepileptiform properties in the $\mathrm{Mg}^{2+}$-free model, ${ }^{8}$ whereby CBDV (at 10 and $100 \mu \mathrm{M}$ ) significantly reduced burst amplitude and duration in the CA1 area of the hippocampus, where such measures are most indicative of drug effects in this system. ${ }^{37}$ In those experiments, 30 min after epileptiform activity commenced, CBDV was applied and allowed to equilibrate for a further $30 \mathrm{~min}$ before recordings were made. For the experiments described here, CBDV was instead added acutely (10 min exposure) and produced similar effects. These data, therefore, confirm and extend our previous
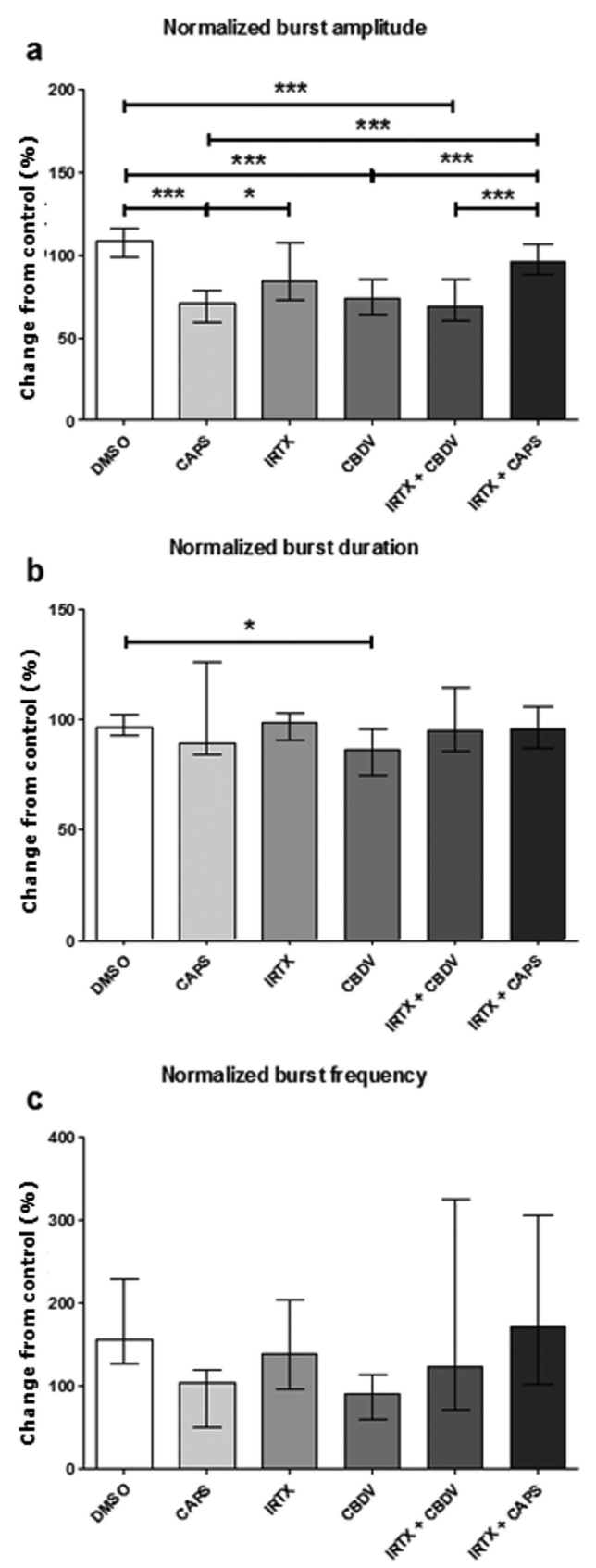

Figure 7. Effects of TRPV1 ligands and CBDV on epileptiform burst amplitude, duration, and frequency in acute hippocampus CA1. Epileptiform activity was induced by application of $\mathrm{Mg}^{2+}$-free ACSF solution for 30-40 min before control recordings were taken. Drugs were applied in the presence of $\mathrm{Mg}^{2+}$-free solution, and recordings taken after $1 \mathrm{~min}$. Effects of vehicle (DMSO), TRPV1 agonist capsaicin (CAPS; $10 \mu \mathrm{M} ; n=25$ electrodes from 4 slices from 2 rats), selective TRPV1 antagonist iodoresiniferatoxin (IRTX; $1 \mu \mathrm{M} ; n=25$ electrodes from 4 slices from 4 rats), CBDV $(10 \mu \mathrm{M} ; n=33$ electrodes from 5 slices from 3 rats), and CBDV or capsaicin in the presence of IRTX ( $10 \mu \mathrm{M}$ and $1 \mu \mathrm{M}$, respectively; $n=34$ electrodes from 5 slices from 3 rats). Neuronal burst (a) amplitude, (b) duration, and (c) frequency are normalized to their own control. Maximum $0.1 \%$ DMSO present. Data represents median with interquartile range and analyzed by Kruskal-Wallis test with Dunn's multiple comparison post hoc $(* p \leq 0.05 ; * * p \leq 0.01 ; * * * \leq 0.001)$.

findings demonstrating that $\mathrm{CBDV}$ is antiepileptiform in the $\mathrm{Mg}^{2+}$-free model. 
Capsaicin, a TRPV1 agonist, also significantly reduced burst amplitude, which we use as the most relevant measure of epileptiform activity in the present study (see ref 37); such antiepileptiform action was antagonized by IRTX, which may reflect activation and desensitization of TRPV1, as per our reported effects in recombinant HEK293 cells above. Thus, the effect of capsaicin might be due to desensitization of endogenously activated TRPV1 channels participating in $\mathrm{Mg}^{2+}$-free-induced neuronal hyperactivity, as assessed by MEA. However, the lack of effect of IRTX on CBDV-induced reduction of burst amplitude seems to rule against this possibility for CBDV. A different approach, described in the next section, was used to determine whether TRPV1 is tonically activated under $\mathrm{Mg}^{2+}$-free conditions in rat hippocampal tissue, and may become desensitized following treatment with capsaicin and CBDV.

CBDV significantly reduced the duration of epileptiform bursts and was, in fact, the only compound to do so with this experimental design. Interestingly, in the presence of IRTX, CBDV effects on burst duration were attenuated. This may suggest that TRPV1 is, in part, implicated in the "duration component" of CBDV's antiepileptiform activity. However, it must be noted that if this was the case then capsaicin would also be expected to reduce burst duration. CBDV has been reported to be a weaker agonist of TRPV1 than capsaicin. ${ }^{14}$ Thus, for now, it is only pertinent to observe that both compounds share one common effect. Further MEA experiments with a range of capsaicin and CBDV concentrations will be useful to reach a definitive conclusion as to whether the effects of capsaicin and CBDV are at least in part mediated by a similar mechanism, and whether such mechanism involves TRPV1 activation/desensitization.

The $\mathrm{Mg}^{2+}$-free model produces spontaneous, synchronous neuronal discharges in acute brain slices that are comparable to the bioelectrical activity observed during status epilepticus. ${ }^{37}$ Despite this, it is important to note that this model is not fully representative of conditions found in an epileptic brain since the neurodegeneration, inflammation, synaptic reorganization, and altered protein expression found in epileptic brain cannot be emulated in acute tissue slices. Here, we focused on the potential effects of CBDV on TRPV1 channels in the $\mathrm{Mg}^{2+}$-free model; in the future, it will be important to extend studies to other TRP isoforms, in particular, TRPV2, and to other in vitro and in vivo seizure models, to more fully explore how the clear effects of phytocannabinoids on TRP activation/desensitization may relate to their anticonvulsant actions.

CBDV Exposure Reduces the Phosphorylation of TRPV1 Channels in the Hippocampus of Epileptic Rats. Changes in TRPV1 expression and/or phosphorylation have been associated with several neurological disorders at both the peripheral and central level. ${ }^{38,39}$ In particular, phosphorylation has been shown to be required for TRPV1 sensitization and cation gating, whereas dephosphorylation is known to desensitize this channel. ${ }^{40}$ TRPV1 has two well-known sites for PKC-mediated phosphorylation: S502 and S800. ${ }^{40} \mathrm{We}$ therefore tested whether the changes in the neuronal activity induced by capsaicin and CBDV, as observed in MEA experiments under our experimental conditions, were associated with changes in TRPV1 phosphorylation at S800. To this purpose, we performed Western blot analysis using anti-TRPV1 and anti-phospho-TRPV1 (pTRPV1) specific antibodies. As shown in Figure 8a, the expression of TRPV1 was not significantly altered in rat hippocampal slices after $1 \mathrm{~h}$ exposure

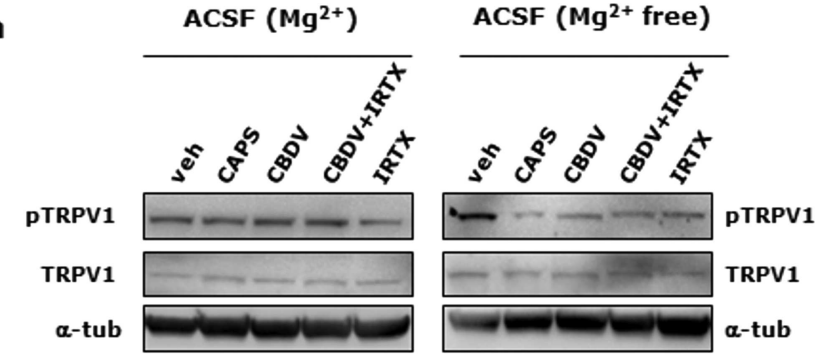

b
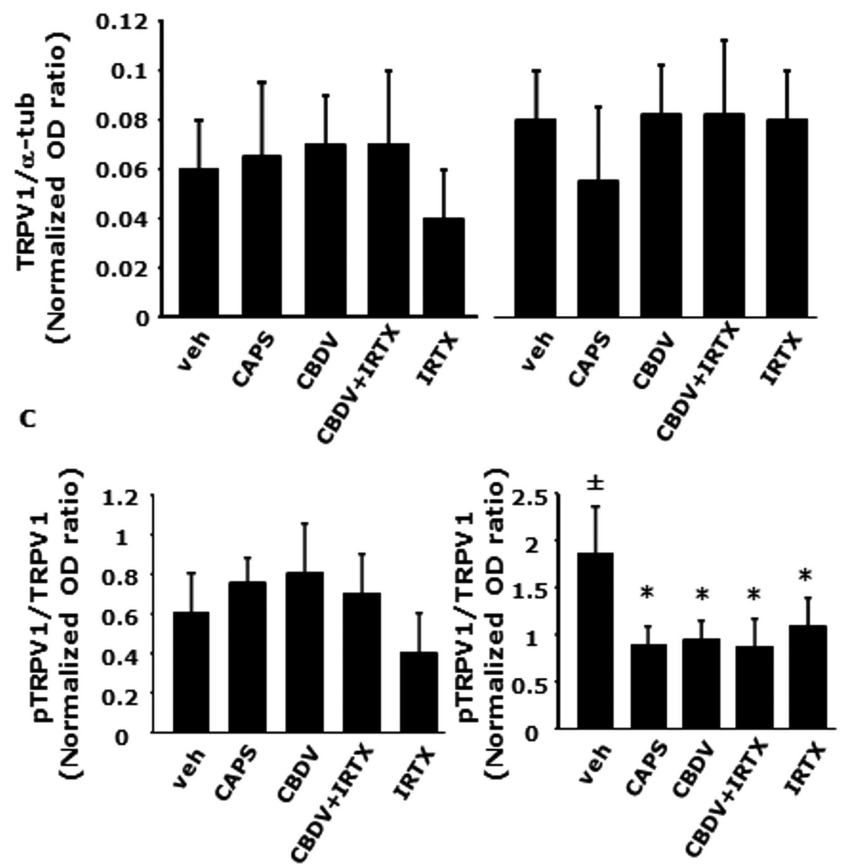

Figure 8. Evaluation of TRPV1 phosphorylation in hippocampal slices upon CBDV exposure TRPV1 protein expression and changes in its phosphorylation at $\mathrm{S} 800$ were evaluated through Western blot analysis of hippocampal slices exposed for about $1 \mathrm{~h}$ to ACSF in the presence (a, control condition) or absence of $\mathrm{Mg}^{2+}$ (b, epileptic-like condition), and either capsaicin (CAPS; $10 \mu \mathrm{M}$ ); iodoresiniferatoxin (IRTX; 1 $\mu \mathrm{M})$; CBDV $(10 \mu \mathrm{M})$ or CBDV $+1 \mu \mathrm{M}$ IRTX. (b) Bar graph showing the quantification of TRPV1 levels normalized to $\alpha$-tubulin. (c) Quantification of phospho-TRPV1 (pTRPV1) levels normalized to total TRPV1. ${ }^{*} p \leq 0.05$ vs respective control; data from three separate samples.

to artificial cerebrospinal fluid (ACSF) in the presence (control condition) or absence (epileptiform condition) of $\mathrm{Mg}^{2+}$. Quantitative analysis of TRPV1 and pTRPV1 was determined relative to the corresponding levels of $\alpha$-tubulin and TRPV1, respectively (Figure $8 \mathrm{~b}$ and $\mathrm{c}$ ). In control slices, we found that $10 \mu \mathrm{M}$ capsaicin or $10 \mu \mathrm{M}$ CBDV, but not $1 \mu \mathrm{M}$ IRTX, tended to increase phosphorylation at the $S 800$ site of TRPV1 (Figure 8 a, left panel), which could suggest the activation of this channel. However, none of the effects reached statistical significance (Figure 8b). On the other hand, in $\mathrm{Mg}^{2+}$-free solutions, we observed a significant increase in the pTRPV1 signal (Figure $8 c$, right panel). These data are consistent with the proposal that neuronal excitability correlates with increased TRPV1 activity. Here, increased excitability may both cause and be caused by TRPV1 activity via increased depolarization. Interestingly, in hippocampal slices exposed to $\mathrm{Mg}^{2+}$ free ACSF, coincubation with capsaicin and CBDV, resulted in a statistically significant reduction of the pTRPV1 signal (Figure 
8c, right panel). In $\mathrm{Mg}^{2+}$-free conditions, IRTX alone also reduced TRPV1 phosphorylation (Figure 8a). The intensity of the band corresponding to total TRPV1 was unchanged (Figure 8a). Quantitative analysis of pTRPV1 expression is reported relative to the corresponding total TRPV1 expression (Figure $8 \mathrm{c}$ ). Collectively, these data suggest that, under conditions in which epileptiform activity is induced in rat hippocampal slices by $\mathrm{Mg}^{2+}$-free solution, a stronger phosphorylation of TRPV1 is observed, which is indicative of TRPV1 sensitization. Under these conditions, both capsaicin and CBDV treatment significantly reduce phosphorylation, thereby potentially leading to TRPV1 desensitization. Thus, despite the fact that our pharmacological data do not support the full involvement of TRPV1 in the action of CBDV in this model, this channel might still participate in the effects of this compound. Furthermore, these data also suggest that CBDV capability of inducing fast TRPV1 desensitization may offer a new opportunity to treat human neurological disorders caused by alteration of TRPV1-mediated conductances, such as epilepsy. However, given the fact that TRPV1 can be modulated by changes in the membrane potential, we cannot rule out the possibility that CBDV and capsaicin-induced TRPV1 dephosphorylation is the consequence, rather than the cause, of CBDV and capsaicin-induced reduction of neuronal hyperexcitability.

\section{CONCLUSIONS}

We demonstrate here that (1) two nonpsychotropic phytocannabinoids, $\mathrm{CBD}$ and $\mathrm{CBDV}$, act as direct agonists and desensitizers of recombinant rat TRPV1, TRPV2, and TRPA1, and that these channels are expressed to a varying extent in rat hippocampal slices; (2) CBDV and capsaicin produce similar, although not identical, effects upon epileptiform activity induced in rat hippocampal slices, as assessed by MEA measurements; (3) capsaicin and CBDV are capable of dephosphorylating rat hippocampal TRPV1 in slices kept in a $\mathrm{Mg}^{2+}$-free solution, thus potentially leading to its desensitization, and to the counteraction of TRPV1 participation in neuronal hyperexcitability. In the future, it will be important to extend the study of phytocannabinoid TRP channel agonists to diverse in vitro and in vivo models of epilepsy.

\section{METHODS}

Cell Culture, Reagents, and Transient Transfection. Human embryonic kidney 293 (HEK293) cells were grown in Dulbecco's modified Eagle's medium supplemented with $10 \%$ fetal bovine serum (FBS), $50 \mathrm{U} / \mathrm{mL}$ penicillin plus $50 \mathrm{mg} / \mathrm{mL}$ streptomycin, and $1 \% \mathrm{~L}$ glutamine in a humidified atmosphere of $95 \% \mathrm{O}_{2} / 5 \% \mathrm{CO}_{2}$ at $37^{\circ} \mathrm{C}$. Cells were transiently transfected with plasmids encoding rat TRPV1, TRPV2, or TRPA1 cDNAs using Lipofectamine LTX (Gibco Invitrogen, Paisley, U.K.) according to the manufacturers' instructions. A plasmid encoding for the enhanced green fluorescent protein (eGFP) was used as a transfection marker.

Patch Clamp Electrophysiology. HEK293 cells transiently expressing rat TRPV1, rat TRPV2, or rat TRPA1 were plated onto poly-L-lysine coated glass coverslips, maintained in $5 \% \mathrm{CO}_{2}$ at $37^{\circ} \mathrm{C}$, and used $24-48 \mathrm{~h}$ after transfection. Recordings from TRP-expressing HEK293 cells were performed using the whole-cell patch clamp technique. Control and test solutions were applied to cells at $0.5 \mathrm{~mL} /$ min via a gravity-fed perfusion system. Recordings were performed at room temperature $\left(20-22{ }^{\circ} \mathrm{C}\right)$. Currents were sampled at $10 \mathrm{kHz}$, amplified, and filtered at $2 \mathrm{kHz}$ using a Digidata 1200 and Axopatch 200B amplifier and analyzed using pClamp 9.0. CBD- or CBDVactivated currents were elicited with a $600 \mathrm{~ms}$ voltage ramp from -80 to $+80 \mathrm{mV}$ at a sampling rate of $10 \mathrm{kHz}$ from a holding potential of -80 or $-100 \mathrm{mV}$. The extracellular solution contained the following (in $\mathrm{mM}$ ): $\mathrm{NaCl}$ (132), $\mathrm{KCl}$ (5.4), $\mathrm{CaCl}_{2}$ (1.8), $\mathrm{MgCl}_{2}$ (0.8), HEPES (10), and glucose (10) at $\mathrm{pH} 7.4$. The pipet solution contained the following (in mM): CsCl (45), CsF (100), EGTA (5), HEPES (10), and glucose (5) at $\mathrm{pH}$ 7.4.

mRNA Extraction and Quantitative PCR (qPCR) Analysis. Total RNA was isolated from native tissues by use of the TRI-Reagent (Life Technology, Milan, Italy), reacted with DNase-I (1U/ $\mu$ L; SigmaAldrich) following the manufacturer's instruction, and then quantified by spectrophotometric analysis. Both sides of the hippocampus were analyzed. Final preparation of RNA was considered DNA- and proteinfree if the ratio between readings at $260 / 280 \mathrm{~nm}$ was $>1.7$. The purified mRNA was reverse-transcribed by use of reverse transcriptase (enzyme-VILO) (Life Technology, Milan, Italy). Quantitative realtime PCR was carried out in CFX384 real-time PCR detection system (Bio-Rad, Segrate MI, Italy) with specific primers (see Supporting Information Table 1) by the use of SYBR Green detection (Bio-Rad, Segrate MI, Italy). Samples were amplified simultaneously in quadruplicate in one assay run with a nontemplate control blank for each primer pair to control for contamination or primer-dimers formation, and the ct (cycle threshold) value for each experimental group was determined. The housekeeping genes (the ribosomal protein S16) has been used as an internal control to normalize the ct values, using the $2^{-\Delta \mathrm{Ct}}$ formula; differences in mRNA content between groups were as expressed as $2^{-\Delta \Delta c t}$, as previously described. ${ }^{41}$

Immunofluorescence. Adult male rats were deeply anesthetized and then perfused/fixed with freshly made paraformaldehyde ( $4 \% \mathrm{w}$ / v) for $5 \mathrm{~min}$. Whole brains were removed from the skull and postfixed in the same fixative for $2 \mathrm{~h}$ at $4{ }^{\circ} \mathrm{C}$, and then transferred to phosphatebuffered saline (PBS, $\mathrm{pH}$ 7.4). Forebrain sagittal vibratome sections $(100 \mu \mathrm{m})$ were cut. Free-floating sections were incubated overnight at $4{ }^{\circ} \mathrm{C}$ with the following primary antibodies: (i) mouse monoclonal anti-TRPV1 antibody (1:100 UC Davis/NIH NeuroMab Facility, CA); (ii) rabbit polyclonal anti-TRPV2 antibody (1:100 Novus Biologicals, LLC); (iii) guinea-pig polyclonal anti-vGLUT1 (iiii) vGAT antibodies (1:250 Synaptic System Inc. Japan). DAPI 1:500 (Sigma-Aldrich, Segrate MI, Italy). All antibodies were diluted in PBS containing $10 \%(\mathrm{v} / \mathrm{v})$ fetal bovine serum and $0.1 \%$ Triton $\mathrm{X}-100$. Slices were then washed in PBS and incubated at $20-22{ }^{\circ} \mathrm{C}$ for $1 \mathrm{~h}$ in a mixture containing two fluorescent secondary antibodies (Alexa flour, Life Tecnologies): (i) chicken anti-mouse or -rabbit 594 for antiTRPV1 and -TRPV2; (ii) donkey anti-guinea pig 488 for anti-vGLUT and -vGAT. Images were acquired by using the digital camera JVC TK-1381 connected to the microscope and the image analysis software AxioVision PlusR 4.0 for Windows (Carl Zeiss, Goettingen, Germany).

Western Blot Analysis. Each animal, previously anesthetized, was decapitated, and the brain quickly removed to prepare hippocampal slices (see section below). The hippocampal slices were exposed to artificial cerebrospinal fluid (ACSF) $\pm \mathrm{MgSO}_{4}$, comprising $(\mathrm{mM})$ $\mathrm{NaCl}$ (124), $\mathrm{KCl}$ (3), $\mathrm{KH}_{2} \mathrm{PO}_{4}$ (1.25), $\mathrm{NaHCO}_{3}$ (36), D-glucose (10), $\mathrm{CaCl}_{2}$ (1) in five distinct conditions: (1) ACSF + DMSO; (2) ACSF + $10 \mu \mathrm{M}$ capsaicin; (3) ACSF $+1 \mu \mathrm{M}$ IRTX; (and 4) ACSF $+10 \mu \mathrm{M}$ CBDV; (5) ACSF $+10 \mu \mathrm{M} \mathrm{CBDV}+1 \mu \mathrm{M}$ IRTX. After this time, the slices were homogenized in lysis solution $(150 \mathrm{mM} \mathrm{NaCl}, 1 \mathrm{mM}$ EDTA, 1\% (v/v) TritonX100, $2.5 \mathrm{mM}$ sodium pyrophosphate, $1 \mathrm{mM}$ b-glycerophosphate, $1 \mathrm{mM} \mathrm{Na}_{3} \mathrm{VO}_{4}, 20 \mathrm{mM}$ Tris-HCl, pH 8, 1\% SDS, plus protease inhibitors) at $\mathrm{pH}$ 7.4. Lysates were boiled for $5 \mathrm{~min}$ in Laemmli SDS loading buffer, loaded $(70 \mu \mathrm{g})$ on $10 \%$ SDSpolyacrylamide gel electrophoresis, and then transferred to a PVDF membrane. Filters were incubated overnight at $4{ }^{\circ} \mathrm{C}$ with the following antibodies: (a) monoclonal anti-TRPV1 clone N221/17 (dilution 1:500 Antibodies Inc.; Davis, CA); (b) polyclonal rabbit antiphospospecific TRPV1 (pTRPV1 S800) (dilution, 1:500; Abnova, Heidelberg Germany). The monoclonal antitubulin clone B-5-1-2 (dilution, 1:5000; Sigma-Aldrich, Milan IT) was used to check for equal protein loading. Reactive bands were detected by chemiluminescence (ECL or ECL-plus; PerkinElmer, Milan IT). Images were analyzed on a ChemiDoc station with Quantity-one software (BioRad, Segrate MI, Italy).

Multielectrode Array Recordings from Acute Hippocampal Brain Slices. Tissue Preparation. The housing and use of animals in 
all experiments were carried out in accordance with U.K. Home office regulations (Animals in Scientific Procedures Act, 1986). Adult male Wistar Kyoto rats (P24-28; Harlan, Bicester, U.K.) were sacrificed by a Schedule 1 method (terminal isoflurane inhalation with death confirmed by cervical dislocation). Each brain was quickly removed and placed immediately in ice-cold, carboxygenated $\left(95 \% \mathrm{O}_{2}: 5 \%\right.$ $\mathrm{CO}_{2}$ ) artificial cerebrospinal fluid (ACSF), comprising (mM) NaCl (124), $\mathrm{KCl}$ (3), $\mathrm{KH}_{2} \mathrm{PO}_{4}$ (1.25), $\mathrm{NaHCO}_{3}$ (36), D-glucose (10), $\mathrm{MgSO}_{4} \cdot 6 \mathrm{H}_{2} \mathrm{O}(1)$, and $\mathrm{CaCl}_{2}$ (2). Transverse hippocampal brain slices $(400 \mu \mathrm{m})$ were taken using a Vibroslice $725 \mathrm{M}$ instrument (Campden Instruments Ltd., Loughborough, U.K.) and stored in carboxygenated ACSF at room temperature for a minimum of $1 \mathrm{~h}$ before use.

Multielectrode Array Recordings. MEA recordings were performed largely as previously described. ${ }^{37}$ Briefly, extracellular neuronal activity in the hippocampus was monitored and recorded using multielectrode arrays (MEAs; 59 electrodes, each $30 \mu \mathrm{m}$ diameter with $200 \mu \mathrm{m}$ spacing; Multi Channel Systems GmbH, Reutlingen, Germany). Prior to recording, MEAs were cleaned with $5 \% \mathrm{w} / \mathrm{v}$ Terg-A-Zyme (ColePalmer, U.K.), rinsed with distilled water, allowed to dry, and gently wiped with a cotton bud dipped in methanol. Hippocampal slices were transferred to MEAs and held in place using a fine mesh anchor to ensure stability and contact with the electrode array. A Nikon TS-51 microscope (Nikon, Japan; magnification $\times 4$ ) was used to carefully position the slice to ensure that the CA1 region lay over the electrode array. Slices were continuously perfused $(\sim 2 \mathrm{~mL} / \mathrm{min})$ with carboxygenated ACSF at $25{ }^{\circ} \mathrm{C}$. Slice viability and contact were assessed by application of biphasic bipolar voltage stimuli $(0.5-1 \mathrm{~V}$; STG2004 stimulator, Multi Channel Systems GmbH, Reulingen, Germany) at electrodes located at the Schaffer collateral pathway to evoke local field potentials in the CA1 region. Signals were amplified (1200× gain), band-pass filtered $(10-3200 \mathrm{~Hz})$ by a 120 -channel dual headstage amplifier (MEA60 system, Multi Channel Systems $\mathrm{GmbH}$, Reutlingen, Germany), and simultaneously sampled at $1000 \mathrm{~Hz}$ on all channels. Data acquisition was to a PC using MC_Rack software (Multi Channel Systems GmbH, Reutlingen, Germany) to monitor and record data for offline analysis.

$\mathrm{Mg}^{2+}$-Free Model of Epileptiform Activity. To induce epileptiform activity, slices were perfused with ACSF with $\mathrm{MgSO}_{4} \cdot 6 \mathrm{H}_{2} \mathrm{O}$ omitted without substitution $\left(\mathrm{Mg}^{2+}\right.$-free ACSF) for 30-40 min. Spontaneous ictal-like bursting activity was then recorded continuously for $10 \mathrm{~min}$. Drugs were applied by dissolving previously prepared aliquots of concentrated stock solutions in the perfused $\mathrm{Mg}^{2+}$-free ACSF. As the TRPV1 receptor is rapidly desensitized following activation, once the drug reached the brain slice $(\sim 1 \mathrm{~min}), 10 \mathrm{~min}$ of recording ictal bursts in the presence of the drug was performed. Drugs used in these experiments were capsaicin (CAPS; $10 \mu \mathrm{M}$; in DMSO; Tocris), iodoresiniferatoxin (IRTX; $1 \mu \mathrm{M}$; in DMSO; Tocris), and cannabidivarin (CBDV; $10 \mu \mathrm{M}$; in DMSO; GW). No more than $0.1 \%$ DMSO was present in the ACSF during experiments. No more than two brain slices from each animal were subjected to the same drug treatment.

Data Analysis. Up to seven electrodes from the CA1 region of each brain slice were selected for analysis; the typical number covering the CA1 region in this slice preparation. For each electrode, the last 10 bursts from each $10 \mathrm{~min}$ recording period were identified using Neuroexplorer (Nex Technologies, Littleton, MA). Using a similar method of analysis as previously described, ${ }^{37}$ the amplitude and duration of each of these 10 bursts were manually measured by playback of data in MC_Rack (Multi Channel Systems GmbH, Reutlingen, Germany). The same 10 bursts were also used to calculate burst frequency where frequency $(\mathrm{Hz})=10 /$ (time of last burst - time of first burst). Individual values for burst amplitude, duration and frequency for each electrode were entered in Microsoft Excel (Microsoft, Redmond, WA) and pooled. Data were then normalized by expressing the drug-treated parameters as a percentage of starting control (where the magnitude of a given measure when no drug was present $=100 \%$ ) and was graphically represented as median with interquartile range. A Kruskal-Wallis one-way analysis of variance with Dunn's multiple comparison posthoc tests was performed using GraphPad Prism (v5, GraphPad Software Inc., La Jolla, CA), and posthoc comparisons conducted using a Mann-Whitney $U$ test. Statistically significant differences were accepted at $p<0.05$.

Reagents. CBD and CBDV (a kind gift of GW Pharmaceuticals Ltd., Salisbury, U.K.) were stored at $-20{ }^{\circ} \mathrm{C}$ as a $100 \mathrm{mM}$ stock solution in dimethyl sulfoxide, which was diluted into the extracellular solution to a final working concentration on the day of use. TRPV1 agonist capsaicin, the TRPV1 antagonists capsazepine and iodoresiniferatoxin (IRTX) (all Tocris Bioscience, Bristol, U.K.), the TRPA1 agonist mustard oil, and the nonselective antagonist ruthenium red (Sigma, Poole, UK) were used.

Other Statistical Analyses. Unless otherwise stated (see MEA section), data were expressed as means \pm SEM of the given number of experiments $(n)$. Data sets were compared by use of matched Student's $t$ tests or, if necessary, with one-way analysis of variance, followed by the Newman-Keul test. Statistically significant differences were accepted at $p<0.05$.

\section{ASSOCIATED CONTENT}

\section{S Supporting Information}

Supplementary Figure 1: Effects of CBDV and CBD in HEK293 cells transiently expressing rat TRPV2 channels. Supplementary Figure 2: Representative MEA traces taken from single electrodes located in the CA1 region of hippocampal slices in control and when exposed to the TRPV1 agonist capsaicin (CAPS; $10 \mu \mathrm{M})$, CBDV $(10 \mu \mathrm{M})$, CBDV in the presence of IRTX $(10 \mu \mathrm{M}$ and $1 \mu \mathrm{M}$, respectively), and CAPS plus IRTX $(10 \mu \mathrm{M}$ and $1 \mu \mathrm{M}$, respectively). Supplementary Table 1: Sequence of primers used for qPCR analysis This material is available free of charge via the Internet at http://pubs.acs.org.

\section{AUTHOR INFORMATION}

\section{Corresponding Authors}

*Mailing address: Endocannabinoid Research Group, Institute of Biomolecular Chemistry (ICB), National Council of Research (CNR), Via Campi Flegrei 34, Comprensorio Olivetti, 80078, Pozzuoli (NA) Italy. Tel: +39-081-8675093. Fax: +39-081-8041770. E-mail: vdimarzo@icb.cnr.it

*Mailing address: School of Pharmacy, University of Reading, Whiteknights, PO Box 228, Reading RG6 6AJ, UK. Tel: +44 118378 6156. Fax: +44-118-3786542. E-mail: g.j.stephens@ reading.ac.uk.

\section{Author Contributions}

F.A.I., G.S., B.W., and V.D. designed research; F.A.I., C.H., E.M., A.L., and C.S. performed research; F.A.I., E.R., C.H., A.L., B.W., and V.D. analyzed data; and F.A.I., G.S., B.W., and V.D. wrote the paper.

\section{Funding}

This work was supported by GW Pharmaceuticals. AL is also supported by students' grants from the European Social Fund and the Calabria Region Government, Italy, who are gratefully acknowledged.

\section{Notes}

The authors declare the following competing financial interest(s): VD acts as a consultant for GW Pharmaceuticals, UK. FAI, BJW, and VD receive research grants from GW Pharmaceuticals, UK.

\section{ACKNOWLEDGMENTS}

We thank Prof. Bernard Attali (Dept of Physiology \& Pharmacology Tel Aviv University, Israel) for kindly providing the TRPV1 plasmid and Dr. Roberta Imperatore for support with microscopy analyses. 


\section{REFERENCES}

(1) Duncan, J. S., Sander, J. W., Sisodiya, S. M., and Walker, M. C. (2006) Adult epilepsy. Lancet 367, 1087-1100.

(2) Barrese, V., Miceli, F., Soldovieri, M. V., Ambrosino, P., Iannotti, F. A., Cilio, M. R., and Taglialatela, M. (2010) Neuronal potassium channel openers in the management of epilepsy: role and potential of retigabine. Clin. Pharmacol. 2, 225-236.

(3) Forsgren, L., Beghi, E., Oun, A., and Sillanpää, M. (2005) The epidemiology of epilepsy in Europe - A systematic review. Eur. J. Neurol. 12 (4), 245-253.

(4) Jones, N. A., Hill, A. J., Smith, I., Bevan, S. A., Williams, C. M., Whalley, B. J., and Stephens, G. J. (2010) Cannabidiol displays antiepileptiform and antiseizure properties in vitro and in vivo. $J$. Pharmacol. Exp. Ther. 332 (2), 569-577.

(5) Jones, N. A., Glyn, S. E., Akiyama, S., Hill, T. D., Hill, A. J., Weston, S. E., Burnett, M. D., Yamasaki, Y., Stephens, G. J., Whalley, B. J., and Williams, C. M. (2012) Cannabidiol exerts anti-convulsant effects in animal models of temporal lobe and partial seizures. Seizure 21 (5), 344-352.

(6) Hill, A. J., Williams, C. M., Whalley, B. J., and Stephens, G. J. (2012) Phytocannabinoids as novel therapeutic agents in CNS disorders. Pharmacol. Ther. 133 (1), 79-97.

(7) Hill, A. J., Mercier, M. S., Hill, T. D., Glyn, S. E., Jones, N. A., Yamasaki, Y., Futamura, T., Duncan, M., Stott, C. G., Stephens, G. J., Williams, C. M., and Whalley, B. J. (2012) Cannabidivarin is anticonvulsant in mouse and rat. Br. J. Pharmacol. 167 (8), 16291642 .

(8) Hill, T. D., Cascio, M. G., Romano, B., Duncan, M., Pertwee, R. G., Williams, C. M., Whalley, B. J., and Hill, A. J. (2013) Cannabidivarin-rich cannabis extracts are anticonvulsant in mouse and rat via a CB1 receptor-independent mechanism. Br. J. Pharmacol. 170 (3), 679-692.

(9) Caterina, M. J., Schumacher, M. A., Tominaga, M., Rosen, T. A., Levine, J. D., and Julius, D. (1997) The capsaicin receptor: a heatactivated ion channel in the pain pathway. Nature 389, 816-824.

(10) Amada, N., Yamasaki, Y., Williams, C. M., and Whalley, B. J. (2013) Cannabidivarin (CBDV) suppresses pentylenetetrazole (PTZ)-induced increases in epilepsy-related gene expression. PeerJ. 21, 1:e214.

(11) Ross, H. R., Napier, I., and Connor, M. (2008) Inhibition of recombinant human T-type calcium channels by Delta9-tetrahydrocannabinol and cannabidiol. J. Biol. Chem. 283 (23), 16124-16134.

(12) Hill, A. J., Jones, N. A., Smith, I., Hill, C., Williams, C., Stephens, G. J., and Whalley, B. J. (2014) Voltage-gated sodium (NaV) channel blockade by plant cannabinoids does not confer anticonvulsant effects per se. Neurosci. Lett. 566, 269-274.

(13) Bisogno, T., Hanus, L., De Petrocellis, L., Tchilibon, S., Ponde, D. E., Brandi, I., Moriello, A. S., Davis, J. B., Mechoulam, R., and Di Marzo, V. (2001) Molecular targets for cannabidiol and its synthetic analogues: Effect on vanilloid VR1 receptors and on the cellular uptake and enzymatic hydrolysis of anandamide. Br. J. Pharmacol. 134 (4), 845-852.

(14) De Petrocellis, L., Vellani, V., Schiano-Moriello, A., Marini, P., Magherini, P. C., Orlando, P., and Di Marzo, V. (2008) Plant-derived cannabinoids modulate the activity of transient receptor potential channels of ankyrin type-1 and melastatin type-8. J. Pharmacol. Exp. Ther. 325 (3), 1007-1015.

(15) De Petrocellis, L., Ligresti, A., Moriello, A. S., Allarà, M., Bisogno, T., Petrosino, S., Stott, C. G., and Di Marzo, V. (2011) Effects of cannabinoids and cannabinoid-enriched Cannabis extracts on TRP channels and endocannabinoid metabolic enzymes. Br. J. Pharmacol. 163 (7), 1479-1494.

(16) Qin, N., Neeper, M. P., Liu, Y., Hutchinson, T. L., Lubin, M. L., and Flores, C. M. (2008) TRPV2 is activated by cannabidiol and mediates CGRP release in cultured rat dorsal root ganglion neurons. J. Neurosci. 28 (24), 6231-6238.

(17) Wong, G. Y., and Gavva, N. R. (2009) Therapeutic potential of vanilloid receptor TRPV1 agonists and antagonists as analgesics: Recent advances and setbacks. Brain Res. Rev. 60 (1), 267-277.
(18) Szallasi, A., and Blumberg, P. M. (1999) Vanilloid (capsaicin) receptors and mechanisms. Pharmacol. Rev. 51 (2), 159-212.

(19) Szallasi, A. (2002) Vanilloid (capsaicin) receptors in health and disease. Am. J. Clin. Pathol. 118, 110-121.

(20) Fernandes, E. S., Fernandes, M. A., and Keeble, J. E. (2012) The functions of TRPA1 and TRPV1: moving away from sensory nerves. Br. J. Pharmacol. 166 (2), 510-521.

(21) Premkumar, L. S., and Bishnoi, M. (2011) Disease-related changes in TRPV1 expression and its implications for drug development. Curr. Top. Med. Chem. 11 (17), 2192-2209.

(22) Cui, M., Honore, P., Zhong, C., Gauvin, D., Mikusa, J., Hernandez, G., Chandran, P., Gomtsyan, A., Brown, B., Bayburt, E. K., Marsh, K., Bianchi, B., McDonald, H., Niforatos, W., Neelands, T. R., Moreland, R. B., Decker, M. W., Lee, C. H., Sullivan, J. P., and Faltynek, C. R. (2006) TRPV1 receptors in the CNS play a key role in broad-spectrum analgesia of TRPV1 antagonists. J. Neurosci. 26 (37), 9385-9393.

(23) Huang, S. M., Bisogno, T., Trevisani, M., Al-Hayani, A., De Petrocellis, L., Fezza, F., Tognetto, M., Petros, T. J., Krey, J. F., Chu, C. J., Miller, J. D., Davies, S. N., Geppetti, P., Walker, J. M., and Di Marzo, V. (2002) An endogenous capsaicin-like substance with high potency at recombinant and native vanilloid VR1 receptors. Proc. Natl. Acad. Sci. U.S.A. 99 (12), 8400-8405.

(24) Cavanaugh, D. J., Chesler, A. T., Jackson, A. C., Sigal, Y. M., Yamanaka, H., Grant, R., O’Donnell, D., Nicoll, R. A., Shah, N. M., Julius, D., and Basbaum, A. I. (2011) TRPV1 reporter mice reveal highly restricted brain distribution and functional expression in arteriolar smooth muscle cells. J. Neurosci. 31 (13), 5067-5077.

(25) Mezey, E., Tóth, Z. E., Cortright, D. N., Arzubi, M. K., Krause, J. E., Elde, R., Guo, A., Blumberg, P. M., and Szallasi, A. (2000) Distribution of mRNA for vanilloid receptor subtype 1 (VR1), and VR1-like immunoreactivity, in the central nervous system of the rat and human. Proc. Natl. Acad. Sci. U.S.A. 97, 3655-3660.

(26) Tóth, A., Boczán, J., Kedei, N., Lizanecz, E., Bagi, Z., Papp, Z., Edes, I., Csiba, L., and Blumberg, P. M. (2005) Expression and distribution of vanilloid receptor 1 (TRPV1) in the adult rat brain. Brain Res. Mol. Brain Res. 135 (1-2), 162-168.

(27) Cristino, L., de Petrocellis, L., Pryce, G., Baker, D., Guglielmotti, V., and Di Marzo, V. (2006) Immunohistochemical localization of cannabinoid type 1 and vanilloid transient receptor potential vanilloid type 1 receptors in the mouse brain. Neuroscience 139, 1405-1415.

(28) Danik, M., Cassoly, E., Manseau, F., Sotty, F., Mouginot, D., and Williams, S. (2005) Frequent coexpression of the vesicular glutamate transporter 1 and 2 genes, as well as coexpression with genes for choline acetyltransferase or glutamic acid decarboxylase in neurons of rat brain. J. Neurosci. Res. 81 (4), 506-521.

(29) Xiong, G., Zhang, L., Mojsilovic-Petrovic, J., Arroyo, E., Elkind, J., Kundu, S., Johnson, B., Smith, C. J., Cohen, N. A., Grady, S. M., and Cohen, A. S. (2012) GABA and glutamate are not colocalized in mossy fiber terminals of developing rodent hippocampus. Brain Res. $1474,40-49$.

(30) Nedungadi, T. P., Dutta, M., Bathina, C. S., Caterina, M. J., and Cunningham, J. T. (2012) Expression and distribution of TRPV2 in rat brain. Exp. Neurol. 237 (1), 223-237.

(31) Shibasaki, K1, Ishizaki, Y., and Mandadi, S. (2013) Astrocytes express functional TRPV2 ion channels. Biochem. Biophys. Res. Commun. 441 (2), 327-332.

(32) Sun, F. J., Guo, W., Zheng, D. H., Zhang, C. Q., Li, S., Liu, S. Y., Yin, Q., Yang, H., and Shu, H. F. (2013) Increased expression of TRPV1 in the cortex and hippocampus from patients with mesial temporal lobe epilepsy. J. Mol. Neurosci. 49 (1), 182-193.

(33) Bhaskaran, M. D., and Smith, B. N. (2010) Effects of TRPV1 activation on synaptic excitation in the dentate gyrus of a mouse model of temporal lobe epilepsy. Exp. Neurol. 223 (2), 529-536.

(34) Shirazi, M1, Izadi, M., Amin, M., Rezvani, M. E., Roohbakhsh, A., and Shamsizadeh, A. (2014) Involvement of central TRPV1 receptors in pentylenetetrazole and amygdala-induced kindling in male rats. Neurol. Sci., DOI: 10.1007/s10072-014-1689-5. 
(35) Kong, W. L., Min, J. W., Liu, Y. L., Li, J. X., He, X. H., and Peng, B. W. (2014) Role of TRPV1 in susceptibility to PTZ-induced seizure following repeated hyperthermia challenges in neonatal mice. Epilepsy Behav. 31, 276-280.

(36) Wahl, P., Foged, C., Tullin, S., and Thomsen, C. (2001) Iodoresiniferatoxin, a new potent vanilloid receptor antagonist. Mol. Pharmacol. 59 (1), 9-15.

(37) Hill, A. J., Jones, N. A., Williams, C. M., Stephens, G. J., and Whalley, B. J. (2010) Development of multi-electrode array screening for anticonvulsants in acute rat brain slices. J. Neurosci Methods. 185 (2), 246-256.

(38) Cortright, D. N., and Szallasi, A. (2004) Biochemical pharmacology of the vanilloid receptor TRPV1. An update. Eur. J. Biochem. 271 (10), 1814-1819.

(39) Planells-Cases, R., Garcia-Sanz, N., Morenilla-Palao, C., and Ferrer-Montiel, A. (2005) Functional aspects and mechanisms of TRPV1 involvement in neurogenic inflammation that leads to thermal hyperalgesia. Pfluegers Arch. 451, 151-159.

(40) Mandadi, S., Tominaga, T., Numazaki, M., Murayama, N., Saito, N., Armati, P. J., Roufogalis, B. D., and Tominaga, M. (2006) Increased sensitivity of desensitized TRPV1 by PMA occurs through PKCepsilon-mediated phosphorylation at S800. Pain 123 (1-2), 106116.

(41) Iannotti, F. A., Piscitelli, F., Martella, A., Mazzarella, E., Allarà, M., Palmieri, V., Parrella, C., Capasso, R., and Di Marzo, V. (2013) Analysis of the "endocannabinoidome" in peripheral tissues of obese Zucker rats. Prostaglandins, Leukotrienes Essent. Fatty Acids 89 (2-3), 127-135. 